\title{
The Role of Constraint and Warm Pre-stress on Brittle Fracture in Ferritic Steels
}

\author{
A.Mirzaee-Sisan, S.Hadidi-moud, C.E. Truman and D.J. Smith \\ Department of Mechanical Engineering, University of Bristol \\ Queen's Building, Bristol, BS8 1TR,UK
}

Keywords: Brittle fracture, failure probability, local approach, triaxiality

\begin{abstract}
This paper presents the results of an experimental and numerical study carried out to investigate the effect of warm pre-stressing on cleavage fracture in ferritic steels using cracked and notched specimens. The local approach based on Weibull assumptions is used to quantify this effect. It is shown that the local approach predicts the increase in toughness following warm pre-stressing in highly constrained geometries. The observed effect of pre-loading in low constraint specimens such as round notched bars is less. The local approach could not predict the differences. It is suggested that the variation of triaxiality factor, which is the ratio of hydrostatic stress to Von Mises, in the plastic zone, was a contributing factor.
\end{abstract}

\section{Introduction}

Warm pre-stressing (WPS) is commonly demonstrated by the Loading-Unloading-CoolFracture (LUCF) load-temperature cycle. It is known that loading and unloading a cracked component at room temperature can enhance its subsequent load bearing capacity at low temperature. Several models to predict the level of improvement in toughness following pre-loading have been developed. Fowler and Smith [1] used a "stress-matching" technique to predict the effect of warm pre-stress. Recently Hadidi-Moud et al [2] applied the "local" approach to predict the effect of warm pre-stress on brittle fracture. In their approach Weibull parameters used in the "local" approach are required. These were calibrated with the aid of finite element analysis using experimental results for as-received (AR) material at low temperature. The same parameters were then used to predict the warm pre-stress effect. The present work follows the same procedure and focuses on attaining an understanding of the role of constraint in the calibration of Weibull parameters and level of improvement in load bearing capacity following warm pre-stressing.

The basis for the "local" approach is the Beremin model [3]. It is assumed that micro-cracks occur at the onset of plastic deformation and that unstable fracture takes place when the maximum principal stress reaches a critical level. In other words, the plastic deformation is referred to as a primary event prior to failure. Global failure is predicted by invoking the weakest link theory. This assumes that a body of material can be fragmented into many independent volumes, linked together like a chain, and the failure of a body commences when its weakest element link fails.

The modified Beremin four-parameter model with inclusion of a minimum threshold stress, $\sigma_{\min }$ 
is given by:

$$
P_{f}\left[\sigma_{f}\right]=1-\exp \left[-\left(\frac{\sigma_{w}-\sigma_{\min }}{\sigma_{u}-\sigma_{\min }}\right)^{m}\right]
$$

where $\sigma_{w}$, the Weibull stress, is:

$$
\sigma_{w}=\left[\sum_{i=1}^{n_{p l}}\left(\sigma_{1}^{i}\right)^{n} \frac{\Delta V_{i}}{V_{0}}\right]^{1 / m}
$$

The Weibull stress, $\sigma_{w}$, is a characteristic stress obtained by integration of the maximum principal stresses over the plastic zone and scaled by a reference volume, $V_{0}$. The reference stress, $\sigma_{u}$, is corresponds to a failure probability of $63.2 \%$ and is referred to as the mean stress. The exponent " $m$ " describes the scatter in the fracture test data. Finally, the threshold stress, $\sigma_{\min }$, is included in the original Beremin model to achieve a better fit to the fracture data.

In the original Beremin approach, the exponent " $m$ " and the reference stress $\sigma_{u}$ were first calibrated using a database of experimental data collected from a wide range of round notched bar (RNB) specimens with various notch tip geometry and tested at different temperatures. It was suggested that the same parameters were appropriate in predicting scatter in fracture data for other configurations including specimens with different geometry and containing sharp cracks. Beremin also suggested that the reference volume, $V_{0}$, was a volume equivalent to about ten grains, typically $V_{0}=0.001 \mathrm{~mm}^{3}$. However, Hadidi-Moud et al [2] has suggested $V_{0}$ is an arbitrary scaling parameter.

The intention in this study is to investigate the dependency of these parameters for specimens with different level of triaxiality (or constraint). In the following sections results from a series of experiments are presented followed by results from finite element studies and detailed probability analysis. To illustrate the different levels of specimen constraint, results are examined in terms of triaxiality factor. The transferability of the Weibull parameters between different specimens with different level of triaxiality are discussed.

\section{Experiments}

Earlier experimental results for twenty eight A533B specimens were obtained by Smith and Garwood [4] using single edge notch bend SEN(B) specimens containing fatigue pre-cracks with nominal $a / W=0.5$ and $W=2 B=100 \mathrm{~mm}$. Fourteen specimens in the as-received condition were loaded to fracture at $-170^{\circ} \mathrm{C}$ with another fourteen in the warm pre-stressed condition at same temperature. The WPS cycle was applied by loading at room temperature equivalent to an elastic stress intensity factor of $120 \mathrm{MPa} \sqrt{\mathrm{m}}$ and then unloading to zero load and cooled to $-170^{\circ} \mathrm{C}$ before being reloaded to fracture.

Recent results for A508 steel using round notched bar (RNB) specimens tested in the AR and WPS conditions at $-150^{\circ} \mathrm{C}$ are summerised here. Thirty four RNB specimens were extracted from a block of A508 Class3 C-Mn steel in the S-L orientation. Sharp (V) and shallow (U) circumferential notches were introduced into the mid section of the specimens. The tip radius of ' $U$ ' shape notches was $1.25 \mathrm{~mm}$, and $0.07 \mathrm{~mm}$ for ' $\mathrm{V}$ ' notches. The sharp notch was introduced by using electrodischarge machining. For the remaining discussion RNB-U1.25 refers to the shallow notch and RNB-V0.07 to the sharp notch. For each notch tip geometry, fracture tests were carried out at $-150^{\circ} \mathrm{C}$ in the as-received and warm pre-stressed conditions. The pre-load level at room temperature 
was equivalent to a net section stress of $994 \mathrm{MPa}$.

The results of SEN(B) in terms of fracture toughness and net section stresses for RNB specimen are shown in against the failure probability in Fig.1 and Fig.2 respectively. The failure probability $P_{f}$ for experimental results was determined using [5]:

$$
P_{f}=\frac{i-0.5}{N}
$$

where $N$ is the total number of specimens, and $i$ the rank or order number.

Using a scanning electron microscope it was observed that all specimens failed by cleavage fracture. The experimental results from the pre-cracked SEN(B) specimens demonstrated that there was a considerable improvement in fracture toughness following WPS. The results from the RNB tests did not show the same level of improvement. The increase in the average fracture stress in RNB-U1.25 was negligible. Nevertheless, the minimum fracture stress for RNB-U1.25 raised from $1231 \mathrm{MPa}$ to $1359 \mathrm{MPa}$. It is noticeable that the RNB-V0.07 specimens show more improvement in fracture stress compared with RNB-U1.25.

\section{Finite Element simulation}

Finite element analyses were performed to provide input data to an analysis that using the local approach to predict failure conditions. The commercial code ABAQUS CAE (V 6.2) was used to create the finite element models. To take advantage of symmetry, one quarter of RNB specimens were modeled using axi-symmetric (CAX8R), iso-parametric quadratic eight noded elements with reduced integration.

For the pre-cracked specimens 3-D models of SEN(B) $W=2 B=100 \mathrm{~mm}$ and a C(T) $W=2 B=50$ $\mathrm{mm}$ were created. All models contained a refined mesh around the crack tip. Two symmetry planes allowed one quarter of the specimens to be modeled, and eight noded quadratic elements with reduced integration $\mathrm{C} 3 \mathrm{D} 8 \mathrm{R}$ were used.

An elastic-plastic material model with isotropic hardening was used for all analyses. For A533B steel the yield strengths $(0.2 \%$ offset $)$ were $528 \mathrm{MPa}$ and $877 \mathrm{MPa}$ at $20^{\circ} \mathrm{C}$ and $-170^{\circ} \mathrm{C}$ respectively. For A508 steel the yield strengths were $430 \mathrm{MPa}$ at $20^{\circ} \mathrm{C}$ and $695 \mathrm{MPa}$ at $-150^{\circ} \mathrm{C}$.

\section{Probability analysis}

\subsection{Calibration of the Weibull parameters}

It was first necessary to calibrate the Weibull parameters, $m, V_{0}, \sigma_{u}$ and $\sigma_{\min }$. To estimate probability of fracture a routine developed by Hadidi-Moud et al [2] was used in conjunction with the results from the FE analyses. A region around the crack/notch tip was chosen as a "fracture process zone" and modeled with a fine mesh in the finite element analysis. This zone was large enough to include all the elements that undergo the plastic deformation during loading to fracture.

The experimental data of pre-cracked specimens carried out by Garwood-Smith [4] were available for $\mathrm{SEN}(\mathrm{B}), \mathrm{B}=50 \mathrm{~mm}$. These data can be converted for use with the $\mathrm{C}(\mathrm{T}), \mathrm{B}=25 \mathrm{~mm}$ model based on the toughness dependence with specimen thickness as follows:

$$
\frac{K_{B 1}}{K_{B 0}}=\left(\frac{B_{0}}{B_{1}}\right)^{1 / 4}
$$


where $K_{B 1}$ and $K_{B 0}$ are the fracture toughness corresponding to $B_{1}$ and $B_{0}$ (the reference thickness).

FE models for both specimens have been analysed. The incremental stress distributions during loading to fracture were used as input for the routine and the Weibull parameters were calibrated using the AR data. Like Hadidi-Moud et al [3] the shape factor $m$ and reference volume were chosen as 4 and $0.01 \mathrm{~mm}^{3}$ respectively. Using either model a good fit was attained by choosing $\sigma_{u}=10.30 \mathrm{GPa}$ and $\sigma_{\min }=3.5 \mathrm{GPa}$. Hence using the same parameters, prediction could be predicted for $\mathrm{SEN}(\mathrm{B}), \mathrm{B}=50 \mathrm{~mm}$ and $\mathrm{C}(\mathrm{T}), \mathrm{B}=25 \mathrm{~mm}$.

The fracture behaviour of RNB specimen was also studied using two different notch tip sharpness. For the RNB-U1.25 the calculation of Weibull stress was performed using the parameters proposed by ESIS [6]. Good agreement was not found between the experiments and predictions. Consequently second attempt was made for the calibration the parameters ( $m, \sigma_{u}$ and $\left.\sigma_{\min }\right)$ and choosing a reference volume as $0.001 \mathrm{~mm}^{3}$. The parameters that provide the best agreement with experimental results at $-150^{\circ} \mathrm{C}$ were $m=24, \sigma_{u}=3.16 \mathrm{GPa}$ and $\sigma_{\min }=0.8 \mathrm{GPa}$. Using these values, the probability of fracture at $-150{ }^{\circ} \mathrm{C}$ shown in Fig 2 was obtained. The same procedure was repeated for RNB-V0.07 and the best agreement with the experimental results was achieved for $m=8, \sigma_{u}=4.98 G P a$ and $\sigma_{\min }=0.0 G P a$ as shown in Fig 2 .

The sets of Weibull parameters were then used to predict the enhancement in cleavage fracture stress following the LUCF cycle for both temperatures.

\subsection{Prediction of the Warm pre-stress}

The WPS was simulated by loading and unloading the FE model using the elastic-plastic material properties at room temperature. This was followed by reloading to fracture using the lower temperature elastic-plastic mechanical properties.

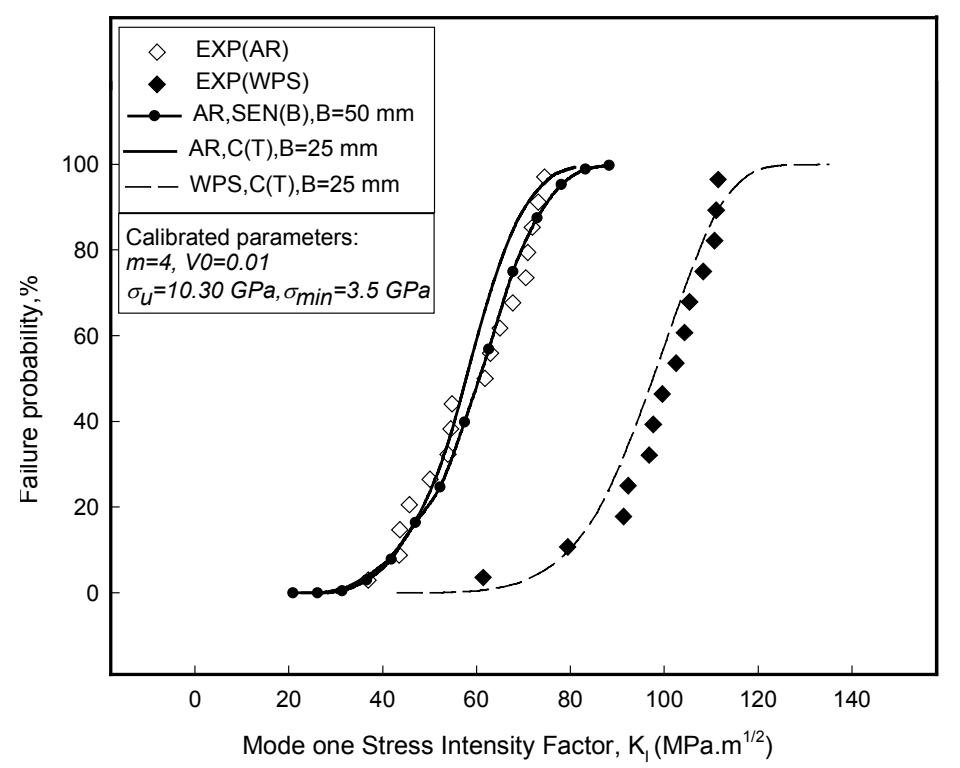

Fig.1. Prediction of failure probability for as-received and warm pre-stressed conditions for A533B steel

For the pre-cracked specimens only $\mathrm{C}(\mathrm{T}), \mathrm{B}=25 \mathrm{~mm}$ model and the "local" routine [2] were used together with the calibrated Weibull parameters to the as-received fracture behaviour, to predict the WPS effect on fracture toughness. Both RNB-U1.25 and RNB-V0.07 models were analysed for the prediction of WPS effect on fracture stress. For pre-cracked specimens the local 
approach predicted significant improvement in toughness following proof loading and a good agreement found between the prediction of the local approach and the experiments.

The results from the WPS simulations for A508 steel RNB specimens are shown in Figure 2. For RNB-U1.25 the simulation predicted an improvement in the fracture stress at low stresses whereas the average of fracture stress remained unchanged. The model also predicted improvements in fracture stress following WPS for RNB-V0.07. However the predicted enhancement was not as much as the experiments suggested.
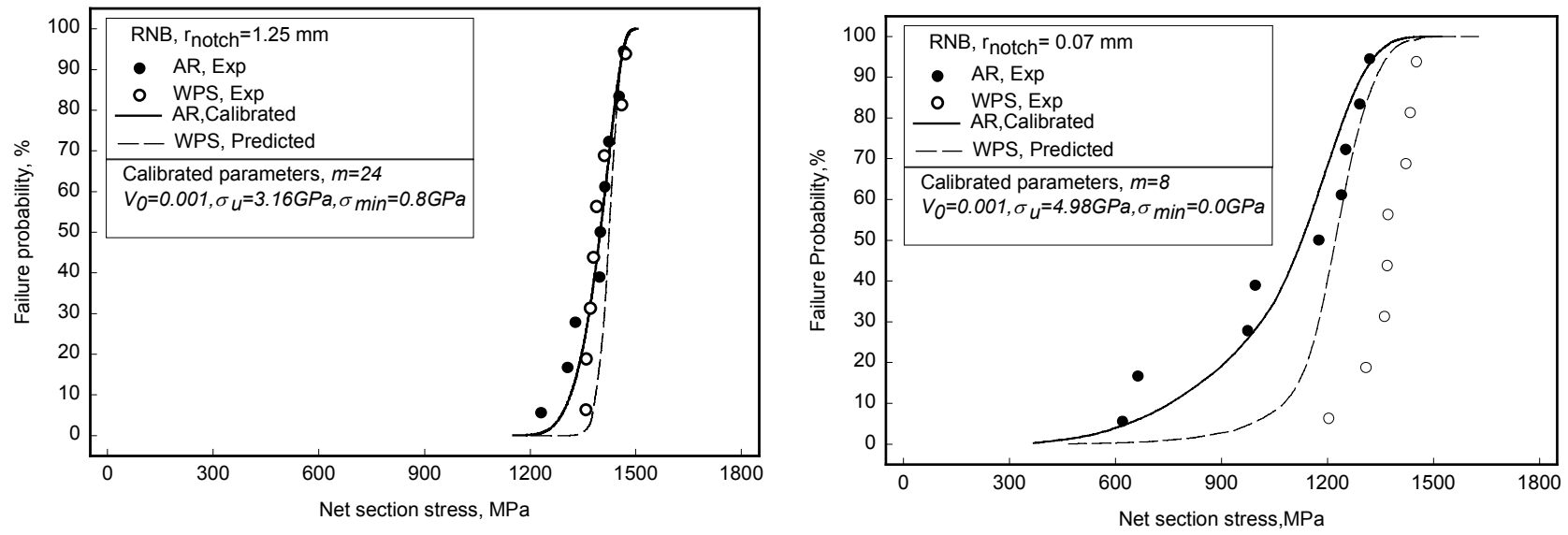

Fig.2. Prediction of failure probability for as-received and warm pre-stressed conditions for A508:

RNB-U1.25 (left); RNB-V0.07 (right)

\section{The Role of constraint}

Milella and Bonora [7] argued that a change in triaxiality would have an impact on the value of $m$ since localised triaxiality determines the extent of the process zone. The triaxiality factor $T_{f}$ is given by the ratio of hydrostatic stress to Von Mises equivalent stress:

$$
T_{f}=\frac{\sqrt{2}}{3} \frac{\sigma_{1}+\sigma_{2}+\sigma_{3}}{\left[\left(\sigma_{1}-\sigma_{2}\right)+\left(\sigma_{2}-\sigma_{3}\right)+\left(\sigma_{1}-\sigma_{3}\right)\right]^{\frac{1}{2}}}
$$

The relationship between $m$ and $T_{f}$ was obtained from experiments is given by:

$$
m=55.4-22.4\left(T_{f}\right)
$$

Here the triaxiality factor has been used as an indicator of the level of constraint between different specimens. Figure 3 shows the incremental variation of triaxiality factor in the plastic zone during loading to fracture in as-received condition. The Weibull stresses were calculated based on integration of principal stress within the plastic zone. Similarly the variation in triaxiality factor was also calculated in the plastic zone. The triaxiality factors in pre-cracked specimens including $C(T)$, $\mathrm{B}=25$ and $\mathrm{SEN}(\mathrm{B}), \mathrm{B}=50$ are very close to each other. Therefore the same value of $m$ was used for all. The magnitude of the calibrated $m$ and the level of triaxiality appeared to satisfy equation 5 .

The triaxiality factor in RNB-U1.25 is lower than RNB-V0.07 and for both RNB specimens less than pre-cracked specimens. Furthermore finite element analysis also showed that the whole net 
section of round notched bar undergoes plastic deformation prior to fracture. This implies that the effect of WPS is wiped out in the presence of large plastic deformation, which was the case for RNB specimens with low constraint. It is noted that the calibrated and predicted $m$ values using Bonora and Millela [7] are in fairly good agreement in RNB and pre-cracked specimens.

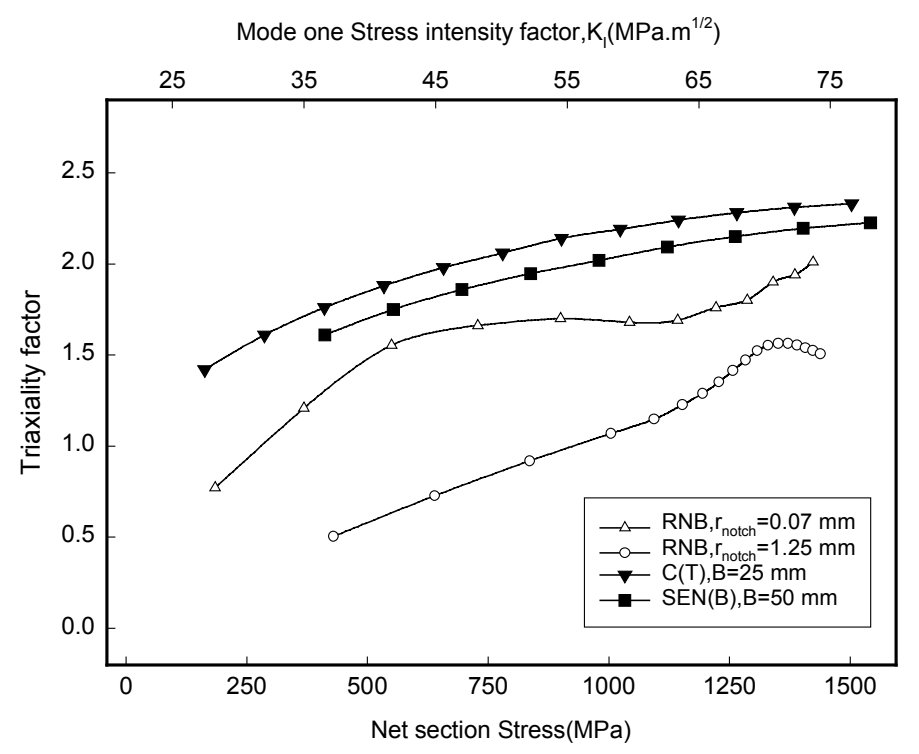

Fig.3. Maximum of triaxiality factors in the plastic zone during loading to fracture in as-received condition

\section{Conclusions}

Hojo et al [8] suggested that the Weibull parameters are independent of geometry. Here we show that the calibrated Weibull parameters for RNB can not be directly applied to highly constrained specimens.

Following LUCF load cycle there is improvement in apparent toughness in highly constrained specimens. Lower triaxiality factors can also explain why the warm pre-stressing does not have a major effect for the RNB specimen.

\section{REFERENCE}

1.Fowler, H., Smith, D.J., SMiRT 14, Lyon, France (1997) p.61

2. Hadidi-Moud S., Mirzaee-Sisan A., Truman C.E. and Smith D.J., PVP Conf., Canada, Vancouver, 434 (2002) p.111

3.Beremin, F.M., J. Metall. Trans. 14A (1983) p. 2277

4.Smith, D.J., Garwood, S.J., Int. J. Pres. Ves. \& Piping, 41 (1990) p.297

5.Bergman, B., J. Mat. Sci. Lett.3 (1984) p.689

6. Catherine C.S., Poussard C., Numerical Round Robin on Micro-Mechanical Models, ESIS

TC 8, (2000)

7.Milella, P.P., Bonora, N., Int. J. Fracture, 104 (2000) p.71

8. Hojo K.,Muroya I., Bruckner-Foit A., Nuclear Eng \& Design, 174 (1997) p.247 